\title{
Limit Theorem on the Sum of Identically Distributed Equally and Positively Correlated Joint Lognormals
}

\author{
Sebastian S. Szyszkowicz, Student Member, IEEE, and Halim Yanikomeroglu, Member, IEEE
}

\begin{abstract}
We prove that the distribution of the sum of $N$ identically distributed jointly lognormal random variables, where all pairs have the same strictly positive correlation coefficient, converges to a lognormal with known parameters as $N$ becomes large. We confirm our theorem by simulations and give an application of the theorem.
\end{abstract}

Index Terms-Sum of lognormals, interference analysis.

\section{INTRODUCTION}

$\mathbf{T}$ HE distribution of the sum of lognormal (SLN) random variables is of interest in wireless communications, since it accurately models the total interference power experienced at a wireless receiver, where individual propagation paths experience lognormal shadowing. Since no closed form is known, finding an approximation to the SLN distribution has been the subject of many papers in wireless communications [1]-[7] (also [8] and additional references therein). Furthermore, this mathematical problem is also of interest in a wide range of other fields, such as economics, microelectronics, photonics, physics, and mathematics (e.g. [9] and some references in [8]).

It can be said that the sum of independent lognormals has received the most attention, as it is a simpler mathematical problem. However, there also exist several papers that deal with approximating the sum of correlated lognormals [3]-[6]. These papers show simulations of a small $(N \leq 24)$ number of correlated lognormal terms. Also, [9] considers $N \leq 50$ correlated terms, but only studies the upper tail of the SLN distribution.

We can argue, however, that these models may not reflect the interference scenarios in future wireless networks. With spectrum-sharing technologies, cognitive radio, use of unlicensed bands, and the proliferation of femtocells, hot-spots, and sensor networks, the number of potential interferers will be several orders of magnitude greater than that in conventional cellular systems. Furthermore, because the shadowing correlation between propagation paths depends on the path lengths and on the angle between them [10], it is expected that the power received from many interferers will suffer from correlated shadowing. As such, it would be useful to analyse the sum of a large number of correlated lognormals.

Paper approved by N. C. Beaulieu, the Editor for Wireless Communication Theory of the IEEE Communications Society. Manuscript received October 16, 2007; revised August 7, 2008.

The authors are with the Broadband Communications and Wireless Systems (BCWS) Centre, Department of Systems and Computer Engineering, Carleton University, Ottawa, K1S 5B6, Canada (e-mail: \{halim, sz $\}$ sce.carleton.ca).

This research was supported in part by a $P G S D$ award from the National Sciences and Engineering Research Council (NSERC) of Canada.

Digital Object Identifier 10.1109/TCOMM.2009.12.070539
Therefore, to our knowledge, for the first time in literature, we study the Sum of Identically Distributed, Equally and (strictly) Positively Correlated Jointly Log-Normal (SIDEPCJLN) random variables as the number of summed terms $N \rightarrow \infty$. In the case of independent lognormals the Central Limit Theorem applies and the distribution is asymptotically Gaussian. There also exist limit theorems where the sums of $(m)$-dependent random variables with finite variance are shown to converge to a Gaussian distribution [11]. However, this is not the case for the SIDEPCJLN, where all terms are correlated with each other. In fact, we will show that for any non-zero correlation coefficient, and for any mean and variance, the limit distribution as $N \rightarrow \infty$ is lognormal. We support this by some Monte Carlo simulations with $N$ large.

It should be noted that there exist only a few papers that give purely closed-form results on the SLN problem [1], [2], [7], [9] and [8] and references therein, and only [9] considers correlated terms. The asymptotic behaviour for large $N$ appears not to have been studied before.

The case of negative correlation is not explored because the problem becomes ill-defined for any $\rho<0$ as $N$ becomes large enough. Indeed, the correlation matrix must always be positive semidefinite [10].

\section{Problem Statement and Motivation}

Let $\vec{W}=\left[W_{i}\right]_{i=1}^{N}$ be a vector of $N$ jointly Gaussian random variables, each with the same mean $\mu$, same variance $\sigma^{2} \neq 0$, and each pair with the same correlation coefficient $0<\rho \leq 1$. Their correlation matrix can thus be written as

$$
\mathbf{K}_{N \times N}=\sigma^{2}\left(\begin{array}{cccc}
1 & \rho & \cdots & \rho \\
\rho & 1 & \cdots & \rho \\
\vdots & \vdots & \ddots & \vdots \\
\rho & \rho & \cdots & 1
\end{array}\right)
$$

Let

$$
Y_{i}=e^{W_{i}} .
$$

We then say that $\vec{Y}=\left[Y_{i}\right]_{i=1}^{N}$ is a jointly lognormal vector, characterised by the same parameters. Now let

$$
X=\sum_{i=1}^{N} Y_{i} .
$$

We say that $X$ follows a SIDEPCJLN distribution with parameters $\mu, \sigma^{2}, \rho, N$. We want to find this distribution, particularly for large $N$.

The results found in this paper are essential to accurately analyse the interference coming from a cluster of many interferers, where the angular separation between the interferers (as seen from the receiver) is small, and hence the shadowing 
terms are highly correlated [10]. Now one might be tempted to argue that the analysis in this paper does not apply, since some interferers are located closer to each other than others, and hence the correlation coefficients are not all equal as in (1). This is true, if one looks at the statistics of the interferers when conditioned upon a particular layout. However, if we consider the interferers as located randomly (within a particular area, not necessarily uniformly), then the problem is perfectly symmetrical and each interferer is, on average, equally correlated with each other one. We explore this idea further in [12], where we successfully predict simulation distribution curves through analysis.

In this paper, we present the fundamental mathematical tool that is necessary to solve the interference problem in [12]. Of course, this result may have applications in other branches of science.

\section{EFFICIENT SimUlation}

In order to generate the Gaussian vector $\vec{W}$ with a given correlation matrix $\mathbf{K}$ and mean vector $\vec{\mu}=\left[\mu_{i}\right]_{i=1}^{N}$, it is necessary to find a "square root" of that matrix, such that

$$
\mathbf{K}=\mathbf{C}^{T} \mathbf{C} \text {. }
$$

This is often done by Cholesky factorisation [10], which results in a triangular matrix. This is a computationally intensive operation for large matrices: for a general matrix, the complexity is $\mathcal{O}\left(N^{3}\right)$ [13]. Having obtained a solution for $\mathbf{C}$, we then generate a vector of independent standard Gaussian random variables $\vec{Z}=\left[Z_{i}\right]_{i=1}^{N}$, from which the vector $\vec{W}$ is calculated as follows:

$$
\vec{W}=\vec{Z} \mathbf{C}+\vec{\mu} .
$$

In general, there are very many solutions to (4), and they are all equally valid for generating $\vec{W}$. In the case of the correlation matrix given in (1), we find that a convenient solution (not the Cholesky factorisation) of (4) is

$$
\mathbf{C}_{N \times N}=\sigma\left(\begin{array}{cccc}
u & v & \cdots & v \\
v & u & \cdots & v \\
\vdots & \vdots & \ddots & \vdots \\
v & v & \cdots & u
\end{array}\right) .
$$

By equating (4), we obtain the system of equations

$$
\left\{\begin{array}{l}
u^{2}+(N-1) v^{2}=1, \\
2 u v+(N-2) v^{2}=\rho,
\end{array}\right.
$$

which has four solutions. We may chose any one of them, for example

$$
\left\{\begin{array}{l}
u_{1}=\frac{1}{N}(\sqrt{1+(N-1) \rho}+(N-1) \sqrt{1-\rho}), \\
v_{1}=\frac{1}{N}(\sqrt{1+(N-1) \rho}-\sqrt{1-\rho}) .
\end{array}\right.
$$

We now have a closed-form expression for the matrix C, which greatly accelerates its computation. However, the memory requirements for this matrix and the computational time for generating $X$ are both $\mathcal{O}\left(N^{2}\right)$, which becomes prohibitive as $N$ increases beyond the order of thousands. We can exploit the particular form of the matrix (6) to compute $X$ more efficiently. Indeed, all entries in each row of the matrix (6) are equal to $v$, except the diagonal terms $u$. Also, all mean parameters are equal: $\mu_{i}=\mu$. We may then write:

$$
\begin{gathered}
Y_{i}=e^{\mu} \exp \left(\sigma v \sum_{j=1}^{N} Z_{j}\right) e^{\sigma(u-v) Z_{i}}, \\
X=e^{\mu} \exp \left(\sigma v \sum_{j=1}^{N} Z_{j}\right) \sum_{i=1}^{N} e^{\sigma(u-v) Z_{i}},
\end{gathered}
$$

where $(u, v)$ is any of the four solutions of (7), for example (8). All four choices will lead to identical distributions for $X$, since any solution of (4) is equally valid to generate $X$ according to (5), (2), and (3).

Therefore $X$ may be directly computed from the vector $\vec{Z}$ using (10), and the time and memory requirements are now $\mathcal{O}(N)$, which allows us to simulate $X$ in reasonable time for $N$ in the order of millions. There exist two alternative methods [14], [15] for generating a correlated Gaussian vector according to (1), also of complexity $\mathcal{O}(N)$.

\section{Limit Theorem AND Moment-Matching}

Theorem. Let $X$ follow a SIDEPCJLN distribution as in (3) with parameters $\mu, \sigma^{2} \neq 0,0<\rho \leq 1, N \in \mathbb{N}^{*}$. Then, as $N \rightarrow \infty$, the quantity $X / N$ tends in distribution to a lognormal random variable with parameters $\left(m_{\infty}, s_{\infty}^{2}\right)$ given by

$$
\begin{aligned}
m_{\infty} & =\mu+\frac{1}{2}(1-\rho) \sigma^{2}, \\
s_{\infty}^{2} & =\rho \sigma^{2} .
\end{aligned}
$$

This theorem is proved in Appendix A. Note that, in the independent case, such as in the Central Limit Theorem, the sum would be normalised by $\sqrt{N}$ because variances would add to each other and the variance of the sum would be $\mathcal{O}(N)$. However, in our correlated case the variance of $X$ is $\mathcal{O}\left(N^{2}\right)$ for $0<\rho \leq 1$ as shown in Appendix B, and thus a normalisation by $N$ is appropriate.

Given that the SIDEPCJLN distribution is lognormal in the limit as $N \rightarrow \infty$, it would be interesting to also examine its behaviour for moderate values of $N$. We reason that since for both $N=1$ and $N \rightarrow \infty$, the SIDEPCJLN distribution is lognormal, then it might be approximately lognormal for intermediate values of $N$. Because a lognormal distribution is uniquely determined by its first and second moments, we can use moment-matching to accurately approximate the SIDEPCJLN distribution, as long as it remains approximately lognormal. We will see in Section $\mathrm{V}$ just how good this approximation is. In Appendix B we will show that $X$ can be approximated in distribution by a lognormal random variable with parameters

$$
\begin{aligned}
& m=\mu+\frac{3}{2} \ln N-\frac{1}{2} \ln \left(1+(N-1) e^{(\rho-1) \sigma^{2}}\right), \\
& s^{2}=\sigma^{2}-\ln N+\ln \left(1+(N-1) e^{(\rho-1) \sigma^{2}}\right) .
\end{aligned}
$$

For high $N$, these expressions simplify to

$$
\begin{aligned}
& m \approx \mu+\ln N+\frac{1}{2}(1-\rho) \sigma^{2}, \\
& s^{2} \approx \rho \sigma^{2} \text { for large } N .
\end{aligned}
$$




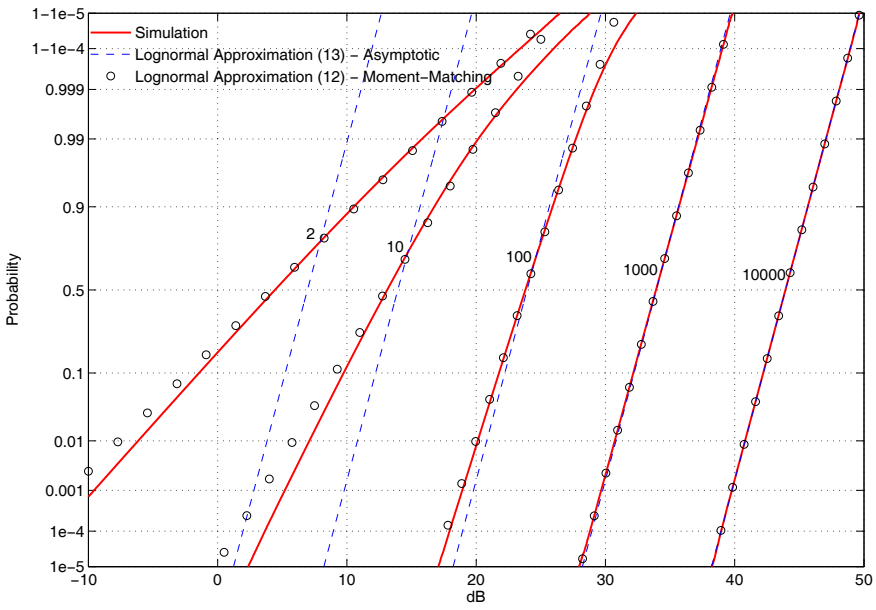

Fig. 1. SIDEPCJLN $c d f$ on lognormal paper, $\sigma=6 \mathrm{~dB}, \rho=0.05$.

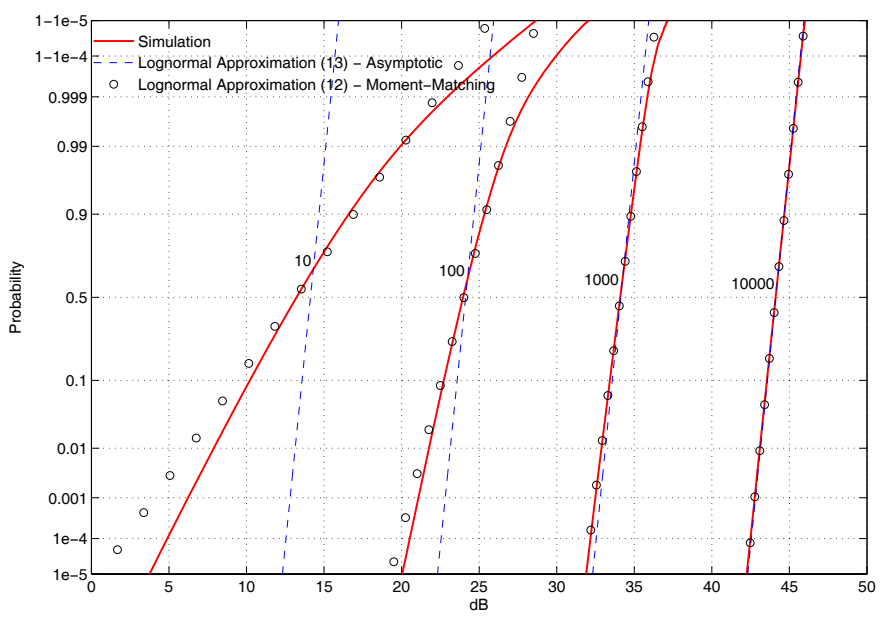

Fig. 2. SIDEPCJLN $c d f$ on lognormal paper, $\sigma=6 \mathrm{~dB}, \rho=0.005$.

Since the distribution of $X$ is approximately lognormal for high $N$ per out theorem, it follows that its lognormal parameters are given by (13), which is consistent with (11), and the additional $\ln N$ term results from the multiplication by $N$.

\section{VAlidation Through Simulation}

Equations (12) and (13) both give the parameters of a lognormal approximation to the SIDEPCJLN distribution. We wish to evaluate the validity of these approximations as a function of the SIDEPCJLN parameters. Since $e^{\mu}$ is a scaling factor, we may set $\mu=0$ without loss of generality. We then fix the parameters $\rho$ and $\sigma$ and increase $N$. We show simulations for $\sigma=6 \mathrm{~dB}$ and $12 \mathrm{~dB}$, and for $\rho=0.05$ and 0.005 . We have also simulated for $\rho=0.5$ (not shown), where the convergence to a lognormal distribution was very fast. Note that for the equations to apply, the value of $\sigma$ must be converted from $\mathrm{dB}$ to natural units as follows: $\sigma[$ nat $]=0.1 \ln 10 \times \sigma[\mathrm{dB}]$. Therefore, $\sigma \cong 1.382$ and 2.763 .

In Figures 1-4, we show Monte Carlo simulations of the SIDEPCJLN distribution computed using (10), and compare them to the lognormals with parameters (12) and (13). We plot the distributions on lognormal paper [8], where lognormal distributions are mapped onto straight lines.

We can make the following observations from these figures:

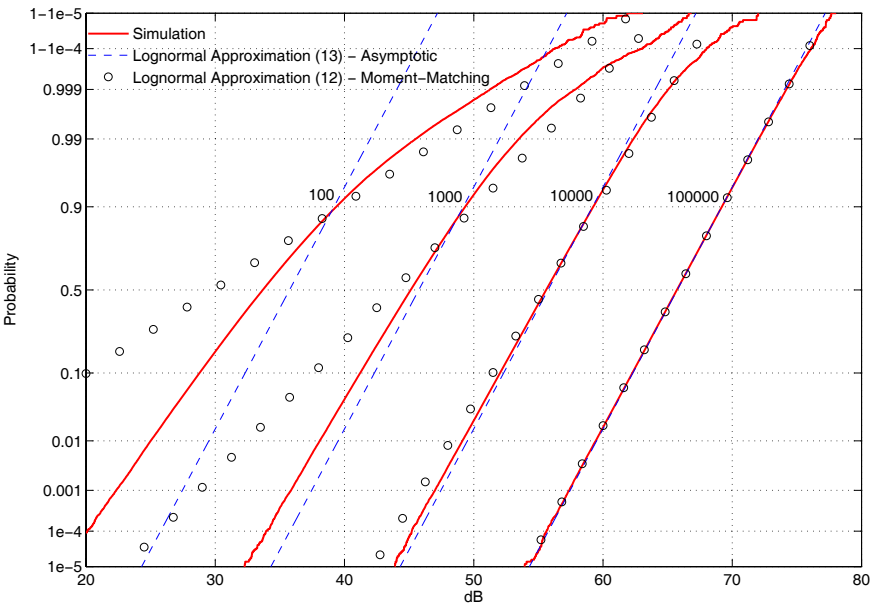

Fig. 3. SIDEPCJLN $c d f$ on lognormal paper, $\sigma=12 \mathrm{~dB}, \rho=0.05$.

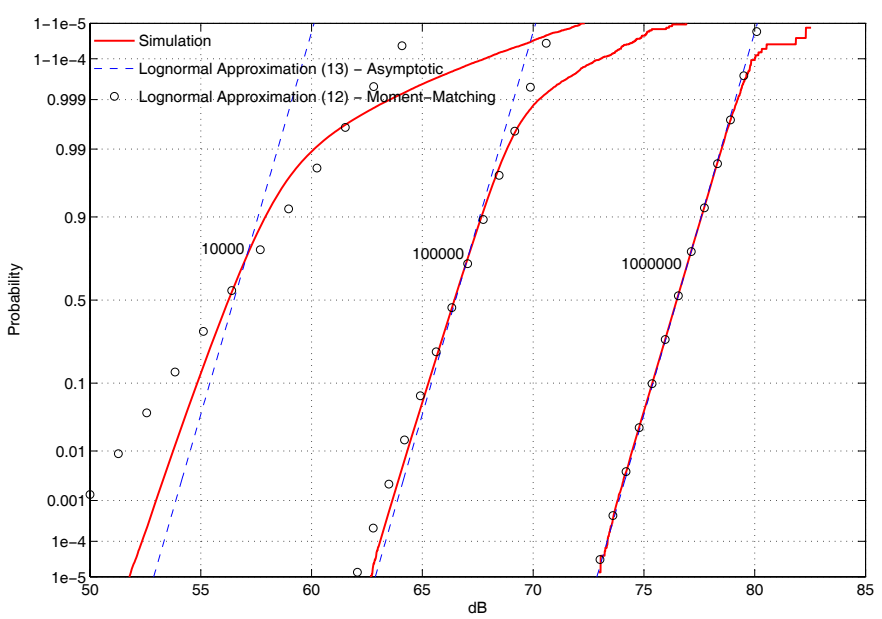

Fig. 4. SIDEPCJLN $c d f$ on lognormal paper, $\sigma=12 \mathrm{~dB}, \rho=0.005$.

1) As per our theorem, for all $0<\rho \leq 1$ and $\sigma^{2} \neq 0$, the SIDEPCJLN distribution tends toward a lognormal distribution with parameters given by (12) or (13) as $N \rightarrow \infty$.

2) The SIDEPCJLN distribution is (trivially) lognormal for $N=1$. For $N \geq 2$, the SIDEPCJLN distribution becomes less and less lognormal until a certain $N$, and then begins to converge back to a lognormal distribution. Once it is again approximately lognormal for $N=N_{0}$, it will remain approximately lognormal for $N \geq N_{0}$.

3) The convergence of the SIDEPCJLN to a lognormal is faster for higher $\rho$, and for lower $\sigma$.

4) Though the SIDEPCJLN distribution converges to a lognormal, this convergence is not uniform over the whole range of values and will in fact never converge at arbitrarily large values, where the behaviour is instead known to be that of the heaviest term in the sum times the number of these heaviest terms, for $-1 \leq \rho<1$ [9] (as long as the matrix remains positive semidefinite). This is notably visible in Figure 4. This is not in contradiction with our result, since we have proved convergence in distribution, i.e., convergence at every point $x$ [16], but not necessarily in the limit behaviour as $x \rightarrow \infty$. 


\section{CONCLUSION}

In this paper we have shown, both through mathematical analysis and Monte Carlo simulations, that the SIDEPCJLN distribution will be well-approximated by a lognormal distribution with parameters (13), when $N$ is large enough. We have observed the convergence of the SIDEPCJLN distribution to this limit and concluded that the convergence is faster for higher correlation coefficient $\rho$ and lower variance $\sigma^{2}$. We have also observed how both the exact (12) and limit (13) moment-matching lognormals approximate the SIDEPCJLN distribution for moderate $N$.

We see that the distribution of $X$ begins as lognormal, then becomes less lognormal (which is well known [4]-[6]), and eventually this trend reverses and we re-enter a lognormal regime. It may be interesting to study how fast $X$ re-enters the lognormal regime (within a certain accuracy, according to some metric), as a function of $\rho$ and $\sigma$. Figures 1-4 already give some idea of this convergence rate.

We present our limit theorem as a general result in mathematics. However, we have also shown in [12] that we may successfully predict with good accuracy the interference distribution from a large cluster of interferers under correlated shadowing by applying our theorem. Indeed, we observe that the interference becomes approximately lognormal for large interfering networks in many realistic scenarios.

\section{Appendix A: Proof of Limit TheOREM}

Proof: In order to prove the theorem, we show that $X / N$ can be written as a product of two terms $A$ and $B$, the first tending in distribution to a constant ${ }^{1}$ as $N \rightarrow \infty$, and the second distributed lognormally. Let

$$
\begin{aligned}
& A=\frac{1}{N} \sum_{i=1}^{N} e^{\sigma(u-v) Z_{i}}, \\
& B=\exp \left(\mu+\sigma v \sum_{j=1}^{N} Z_{j}\right) .
\end{aligned}
$$

We find

$$
(u-v)^{2}=1-\rho
$$

from (7). Then

$$
\begin{aligned}
\mathbb{E}\{A\} & =e^{\frac{1}{2}(1-\rho) \sigma^{2}}, \\
\mathbb{E}\left\{A^{2}\right\} & =\frac{1}{N} e^{2(1-\rho) \sigma^{2}}+\frac{N-1}{N} e^{(1-\rho) \sigma^{2}}, \\
\operatorname{VAR}\{A\} & =\frac{1}{N}\left(e^{2(1-\rho) \sigma^{2}}-e^{(1-\rho) \sigma^{2}}\right) .
\end{aligned}
$$

Since the variance of $A$ tends to zero as $N \rightarrow \infty, A$ tends in distribution to a constant equal to $\mathbb{E}\{A\}$ [16].

Now from (8) we may find the asymptotic behaviour of $v$ :

$$
v \approx \sqrt{\rho / N} \text { for large } N .
$$

A different solution of (7) for $v$ may lead to the same asymptotic expression with a negative sign in front, which does not affect the proof.

\footnotetext{
${ }^{1}$ Convergence to a constant in distribution and in probability are mathematically equivalent [16]. For our purposes here, convergence in distribution is always sufficient.
}

Now $B$ follows a lognormal distribution. From (14) and (17) we find that it has parameters $\left(m_{0}, s_{0}^{2}\right)$ equal to

$$
\begin{aligned}
m_{0} & =\mu, \\
s_{0}^{2} & \approx \rho \sigma^{2} \text { for large } N .
\end{aligned}
$$

This holds only for $0<\rho \leq 1$. If $\rho=0$, then $B$ becomes a constant, and the rest of the argument does not follow.

Finally, we have

$$
X / N=A B .
$$

This quantity is the product of a random variable that tends to a constant in distribution, with a lognormal random variable that tends to a particular lognormal distribution. According to Slutsky's Theorem [16], the product will therefore tend to a lognormal distribution, and its parameters can be calculated from (16), (18) and (19), and are $\left(m_{\infty}, s_{\infty}^{2}\right)$ as given in (11).

However, if $\rho=0$, the quantity $A B$ converges to the constant $e^{\mu+\frac{1}{2} \sigma^{2}}$, as expected from the Law of Large Numbers.

\section{ApPendix B: Moment-Matching Analysis}

The first moment of $X$ is found using (7) as follows:

$$
\begin{aligned}
\mathbb{E}\{X\} & =e^{\mu} \sum_{i=1}^{N} \mathbb{E}\left\{\exp \left(\sigma v \sum_{j=1, j \neq i}^{N} Z_{j}\right)\right\} \mathbb{E}\left\{e^{\sigma u Z_{i}}\right\} \\
& =N e^{\mu+\frac{1}{2} \sigma^{2}\left((N-1) v^{2}+u^{2}\right)}=N e^{\mu+\frac{1}{2} \sigma^{2}} .
\end{aligned}
$$

Similarly, the second moment is found to be

$$
\mathbb{E}\left\{X^{2}\right\}=N e^{2 \mu+2 \sigma^{2}}\left(1+(N-1) e^{(\rho-1) \sigma^{2}}\right) .
$$

The variance of $X$ is therefore

$$
\begin{aligned}
\mathbb{V A R}\{X\} & =N^{2} e^{2 \mu+\sigma^{2}}\left(e^{\rho \sigma^{2}}-1\right) \\
& +N e^{2 \mu+2 \sigma^{2}}\left(1-e^{(\rho-1) \sigma^{2}}\right) .
\end{aligned}
$$

Equating these moments to those of a lognormal random variable $\tilde{X}$ with parameters $\left(m, s^{2}\right)$ yields

$$
\begin{aligned}
m & =2 \ln \mathbb{E}\{\tilde{X}\}-\frac{1}{2} \ln \mathbb{E}\left\{\tilde{X}^{2}\right\} \\
& =2 \ln \mathbb{E}\{X\}-\frac{1}{2} \ln \mathbb{E}\left\{X^{2}\right\}, \\
s^{2} & =-2 \ln \mathbb{E}\{\tilde{X}\}+\ln \mathbb{E}\left\{\tilde{X}^{2}\right\} \\
& =-2 \ln \mathbb{E}\{X\}+\ln \mathbb{E}\left\{X^{2}\right\},
\end{aligned}
$$

which gives (12), after substituting (20) and (21).

\section{ACKNOWLEDGMENT}

The authors would like to thank Dr. Miklós Csörgó (Dept. of Mathematics and Statistics, Carleton University, Canada), Dr. Norman C. Beaulieu (University of Alberta, Canada), and Dr. John S. Thompson (University of Edinburgh, United Kingdom) for valuable discussions. 


\section{REFERENCES}

[1] F. Berggren and S. Ben Slimane, "A simple bound on the outage probability with lognormally distributed interferers," IEEE Commun. Lett., vol. 8, pp. 271-273, May 2004.

[2] F. Berggren, "An error bound for moment matching methods of lognormal sum distributions," European Trans. Telecommun., vol. 16, pp. 573-577, 2005.

[3] A. A. Abu-Dayya and N. C. Beaulieu, "Outage probabilities in the presence of correlated lognormal interferers," IEEE Trans. Veh. Technol., vol. 43, pp. 164-173, Feb. 1994.

[4] P. Pirinen, "Statistical power sum analysis for nonidentically distributed correlated lognormal signals," in Proc. Finnish Signal Processing Symp. (FINSIG), pp. 254-258, May 2003.

[5] C. L. J. Lam and T. Le-Ngoc, "Outage probability with correlated lognormal interferers using log shifted gamma approximation," in Proc. International Conf. Inf., Commun. Signal Processing (ICICS), pp. 618622, Dec. 2005.

[6] N. B. Mehta, A. F. Molisch, J. Wu, and J. Zhang, "Approximating the sum of correlated lognormal or, lognormal-Rice random variables," in Proc. IEEE International Conf. Commun. (ICC), vol. 4, pp. 1605-1610, June 2006.

[7] H. Nie and S. Chen, "Lognormal sum approximation with type IV Pearson distribution," IEEE Commun. Lett., vol. 11, pp. 790-792, Oct. 2007.
[8] S. S. Szyszkowicz and H. Yanikomeroglu, "On the tails of the distribution of the sum of lognormals," in Proc. IEEE International Conf. Commun. (ICC), pp. 5324-5329, June 2007.

[9] S. Asmussen and L. Rojas-Nandayapa, "Asymptotics of sums of lognormal random variables with Gaussian copula," Statistics Probability Lett., vol. 78, pp. 2709-2714, Nov. 2008.

[10] T. Klingenbrunn and P. Mogensen, "Modelling cross-correlated shadowing in network simulations," in Proc. IEEE Veh. Technol. Conf. (VTC), vol. 3, pp. 1407-1411, Sep. 1999.

[11] W. Hoeffding and H. Robbins, "The central limit theorem for dependent random variables," Duke Mathematical J., vol. 15, pp. 773-780, 1948.

[12] S. S. Szyszkowicz and H. Yanikomeroglu, "Analysis of interference from large clusters as modeled by the sum of many correlated lognormals," in Proc. IEEE Wireless Commun. Netw. Conf., pp. 741-745, Mar. 2008.

[13] B. Alkire, "Cholesky factorization of augmented positive definite matrices," Electrical Engineering Department, UCLA, Dec. 2002.

[14] A. J. Coulson, A. G. Williamson, and R. G. Vaughan, "A statistical basis for lognormal shadowing effects in multipath fading channels," IEEE Trans. Commun., vol. 46, pp. 494-502, Apr. 1998.

[15] R. Fraile, J. F. Monserrat, J. Gozálvez, and N. Cardona, "Mobile radio bi-dimensional large-scale fading modelling with site-to-site crosscorrelation," European Trans. Telecommun., vol. 19, pp. 101-106, Jan. 2008.

[16] V. K. Rohatgi and A. K. M. E. Saleh, An Introduction to Probability and Statistics, 2nd ed. Wiley-Interscience, 2001. 